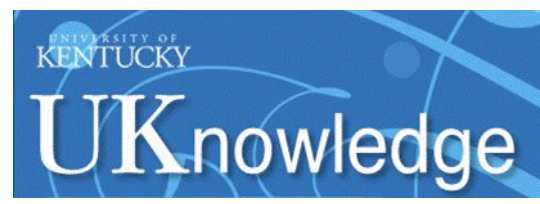

University of Kentucky

UKnowledge

$11-15-1982$

\title{
Broad Line Region Clouds and the Absorbing Material in NGC
}

\section{1}

Gary J. Ferland

University of Kentucky, gary@uky.edu

R. F. Mushotzky

NASA Goddard Space Flight Center

Follow this and additional works at: https://uknowledge.uky.edu/physastron_facpub

Part of the Astrophysics and Astronomy Commons, and the Physics Commons

Right click to open a feedback form in a new tab to let us know how this document benefits you.

\section{Repository Citation}

Ferland, Gary J. and Mushotzky, R. F., "Broad Line Region Clouds and the Absorbing Material in NGC 4151" (1982). Physics and Astronomy Faculty Publications. 183.

https://uknowledge.uky.edu/physastron_facpub/183

This Article is brought to you for free and open access by the Physics and Astronomy at UKnowledge. It has been accepted for inclusion in Physics and Astronomy Faculty Publications by an authorized administrator of UKnowledge. For more information, please contact UKnowledge@lsv.uky.edu. 


\section{Broad Line Region Clouds and the Absorbing Material in NGC 4151}

Digital Object Identifier (DOI)

http://dx.doi.org/10.1086/160448

\section{Notes/Citation Information}

Published in The Astrophysical Journal, v. 262, no. 2, p. 564-577.

(c) 1982. The American Astronomical Society. All rights reserved.

The copyright holder has granted permission for posting the article here. 
The Astrophysical Journal, 262:564-577, 1982 November 15

( 1982 . The American Astronomical Society. All rights reserved. Printed in U.S.A.

BROAD LINE REGION CLOUDS AND THE ABSORBING MATERIAL IN NGC 4151

\author{
G. J. FERLAND \\ Department of Physics and Astronomy, University of Kentucky, Lexington \\ AND \\ R. F. MUSHOTZKY \\ Laboratory for High Energy Astrophysics, NASA Goddard Space Flight Center \\ Received 1981 December 28; accepted 1982 April 26
}

\begin{abstract}
The constraints imposed by X-ray, ultraviolet, and optical observations on the absorbing material in the Seyfert 1.5 galaxy NGC 4151 are discussed. These observations are compared to the predictions of photoionization models of both broad line region clouds, which Holt et al. identify as the source of the extinction, and to models of the narrow line region gas. The weakness of both C III] 1909 and the broad component of $\mathrm{H} \alpha$ relative to $\mathrm{C}$ IV 1549, together with the absence of an absorption Balmer jump, indicate that the ionization parameter for the broad line region is a factor $\sim 30$ times larger in NGC 4151 than is found in typical QSO clouds. The energy budget of the system, as deduced from model calculation and emission-line fluxes, demands essentially full coverage of the continuum source by both broad and narrow line region clouds. The continuum incident on the outer narrow line region clouds is dominated by hard $(h \nu>5 \mathrm{keV}) \mathrm{X}$-rays because of the effective low frequency absorption arising in the inner broad line region gas. The calculations suggest that narrow line region clouds have a composition similar to extragalactic giant $\mathrm{H}$ II regions, and the hydrogen line spectrum shows that dust is mixed with narrow line region gas. We argue that broad line region clouds are likely to be in a radiatively driven wind and show that these clouds are opaque to radio emission. Some other consequences of these calculations are also discussed.
\end{abstract}

Subject headings: galaxies: individual - galaxies: Seyfert $-\mathrm{X}$-rays: sources

\section{INTRODUCTION}

One surprising result of ground-based studies of high redshift quasars has been the relative scarcity of objects in which the line of sight passes through emission line region gas (see Smith et al. 1981). Photoionization models (see Baldwin and Netzer 1978; Davidson 1977; Davidson and Netzer 1979) suggest that both the broad line region (BLR) and the narrow line region (NLR) gas should be optically thick at the Lyman limit. The fact that few objects show $\lambda 912 \AA$ absorption at the emission-line redshift apparently indicates that the gas has coalesced into small filaments or blobs and that the covering factor, or fraction of ionizing photons which actually intercept a BLR or NLR cloud, must be small (typically $\varepsilon \sim 0.15$, Smith et al. 1981).

This small covering factor affects several problems associated with emission line spectra. Baldwin (1977a) pointed out that $\varepsilon \sim 0.15$ is consistent with the equivalent width of Ly $\alpha$ in quasars, but not that of the Balmer lines. This so-called $\mathrm{Ly} \alpha / \mathrm{H} \beta$ problem has been largely resolved by recent emission-line models which include the effects of the strong X-ray continuum on the neutral gas beyond the $\mathrm{H}^{+}-\mathrm{H}^{0}$ ionization front (see Kwan and Krolik 1981; Weisheit, Shields, and Tarter 1981). A second question results from Baldwin's (1977b) discovery that the equivalent width of C IV $\lambda 1549$ is correlated with a quasar's luminosity. This correlation may indicate that the covering factor is somehow related to luminosity with luminous objects having the smaller value (although dust may also play a role, see Shuder and MacAlpine 1979). If the C IV equivalent widthluminosity relation is indeed related to the covering factor, then the lowest luminosity objects (e.g., Seyfert galaxies) should have the largest covering factor and stand the best chance of actually having emission line region gas along the line of sight. The identification and study of such cases is important since both line and continuum absorption from emission line region gas can provide valuable information available in no other way (see Peterson et al. 1982).

In this paper we reexamine the observational evidence concerning the obscuring material along the line of sight to the continuum source in the Seyfert 1.5 galaxy NGC 4151 (Netzer 1974; Osterbrock 1977). Holt et al. (1980) have argued that this absorption arises in a large number of BLR clouds. We discuss the implications of both the soft X-ray continuous extinction and the optical and ultraviolet absorption lines (cf. Penston et al. 1981) by 
comparing these data with predictions of a grid of photoionization models. In the next section we outline the basic model of emission line formation in active galactic nuclei and discuss some characteristics of the absorbing material in NGC 4151. In $\S$ III we compile a mean emission-line spectrum and model both the narrow and broad line regions. Some implications of these models are discussed both there and in the last section. The need for more observations, particularly of the poorly monitored optical spectrum, is stressed.

\section{THE ABSORBING MATERIAL}

The constraints imposed by optical, ultraviolet, and $\mathrm{X}$-ray observations on the nature of the absorbing material in NGC 4151 are discussed in this section.

\section{a) The Basic Model}

Emission-line regions of active galactic nuclei (AGNs) are usually modeled in terms of an ensemble of filaments or blobs in photoionization equilibrium with the radiation field of the central object (see Davidson 1972; MacAlpine 1972). Two distinct emission-line regions are usually identified; an inner broad line region characterized by a density $N \sim 10^{9}-10^{10} \mathrm{~cm}^{-3}$, radius $\sim 10^{17}$ $\mathrm{cm}$, and line width corresponding to velocities $\sim 5000$ $10,000 \mathrm{~km} \mathrm{~s}^{-1}$, together with an outer narrow line region $\left(N \sim 10^{3}-10^{6} \mathrm{~cm}^{-3}, R \sim 10^{19} \mathrm{~cm}, V \sim 500-1000\right.$ $\mathrm{km} \mathrm{s}^{-1}$, see Shields 1974). The BLR clouds are generally thought to be in pressure equilibrium with a hot, lowdensity medium which pervades this inner region (see Fabian and Ross 1981; Shields and McKee 1981; Krolik, McKee, and Tarter 1981). The nature of the motions leading to the large line widths is uncertain; radiatively driven outflow may be a natural consequence of the physical environment (see Blumenthal and Mathews 1979), although we shall see that absorption variability in NGC 4151 suggests that a large transverse component to the velocity is also present. The large emissionline widths are generally thought to be indicative of relative motions of individual clouds, since the importance of line trapping phenomena suggests that motions within a particular cloud are largely thermal (see Davidson and Netzer 1979). The physical sizes of individual clouds are uncertain, but a variety of arguments suggest $\delta r<10^{15} \mathrm{~cm}$ for quasars (Carswell and Ferland 1980), and Holt et al. (1980) argue that $\delta r \sim 10^{13}$ $\mathrm{cm}$ for NGC 4151 .

The continuum presumed to heat and ionize these clouds in NGC 4151 has been well studied over a wide range of energies. Like that of most AGNs, the continuum is well approximated by two power laws (see Wilson 1979; Mushotzky et al. 1980). The optical to ultraviolet continuum is close to

$$
F_{\nu}=1.1 \times 10^{28}\left(\frac{\nu}{1.5 \times 10^{15} \mathrm{~Hz}}\right)^{-1.5} \operatorname{ergs~s}^{-1} \mathrm{~Hz}^{-1}
$$

(Boksenberg et al. 1975; Penston et al. 1981; we assume a distance of $20 \mathrm{Mpc}$ ), while the X-ray to $\gamma$-ray continuum is much flatter:

$$
F_{\nu}=1.5 \times 10^{26}\left(\frac{\nu}{1.5 \times 10^{15} \mathrm{~Hz}}\right)^{-0.5} \operatorname{ergs~s}^{-1} \mathrm{~Hz}^{-1}
$$

(see Mushotzky, Holt, and Serlemitsos 1978). Both continua are variable with time scales of $\sim 1$ day in the $\mathrm{X}$-ray, corresponding to a light travel time radius $R \sim$ $10^{15.5} \mathrm{~cm}$ (Mushotzky, Holt, and Serlemitsos 1978).

\section{b) X-Ray Observations}

NGC 4151 is a low-luminosity $\left(L_{x} \sim 10^{42.5} \mathrm{ergs} \mathrm{s}^{-1}\right.$, 2-10 keV) X-ray source which has been well studied because of its large apparent luminosity, intrinsic variability (see Mushotzky, Holt, and Serlemitsos 1978), and low-frequency cutoff (Barr et al. 1977, Holt et al. 1980). Holt et al. found that the $0.5-5 \mathrm{keV}$ continuum had been extinguished by a leaky absorber covering 94\% of the source with a column density of $N_{\mathrm{H}}=6.1 \times 10^{22}$ $\mathrm{cm}^{-2}$. They argue that the projected continuum source is covered by $\sim 10^{2}$ small $\left(\delta r \sim 10^{13} \mathrm{~cm}\right)$ clouds and that roughly two clouds lie along a typical line of sight. In this picture $\sim 10 \%$ of the source will be uncovered because of statistical fluctuations in placement of the clouds. The fact that the apparent column density changes over a time scale of about 1 year (varying from $\sim 3-15 \times 10^{22} \mathrm{~cm}^{-2}$; Barr et al. 1977) thus suggests that absorbing clouds are able to drift across the continuum source within this time and implies a transverse velocity of $\sim 10^{3} \mathrm{~km} \mathrm{~s}^{-1}$. The conclusion that the absorbing material is BLR gas follows from both this velocity and the fact that the position of the observed Fe K-edge indicates that iron is cold (i.e., has more than $\sim 14$ electrons; Holt et al. 1980). This suggestion is also in agreement with model calculations which show that BLR clouds are capable of providing the needed extinction of soft X-rays (see Shields and Mushotzky 1979; Weisheit, Shields, and Tarter 1981).

\section{c) Absorption Lines}

In addition to the X-ray low-frequency cutoff described above, absorbing material is also evidenced as a set of ultraviolet resonance absorption lines (e.g., C IV $\lambda 1549, \mathrm{Mg}$ II $\lambda 2798$; see Penston et al. 1979 , Penston et al. 1981) and optical subordinate lines (He I $\lambda 3889$, the Balmer lines; see Anderson and Kraft 1969; Anderson 1974; and Cromwell and Weymann 1970). Both the ionization species present and the radial velocities relative to the rest frequency, typically $\sim 10^{3}$ $\mathrm{km} \mathrm{s}^{-1}$, suggest that these lines arise in BLR clouds. These clouds must be undergoing outflow since absorption components have always tended to lie shortward of the rest wavelength (see Anderson and Kraft 1969; Penston et al. 1979). 
A surprising result of these studies is the large breadth of absorption features. Both the optical and highresolution ultraviolet data show that these lines have widths of typically $\sim 300-1000 \mathrm{~km} \mathrm{~s}^{-1}$ (Penston et al. 1979; Anderson 1974). Since BLR clouds are generally thought to be in pressure equilibrium (see Davidson and Netzer 1979; Krolik, McKee, and Tarter 1981), they should be characterized by largely thermal $\left(\sim 10 \mathrm{~km} \mathrm{~s}^{-1}\right.$ for hydrogen) line widths. The large line widths cannot plausibly be ascribed to microturbulence within a single cloud for several reasons. First, the widths correspond to shocks with Mach numbers $\sim 30-100$. Such shocks would heat the gas to $\sim 10^{6.6}-10^{7} \mathrm{~K}$ and lines due to $\mathrm{C}$ IV, $\mathrm{Mg}$ II, and $\mathrm{H}$ I would not be present. It can also be shown that resonance line trapping would be relatively unimportant for such line widths, and absorption from excited states (such as the Balmer lines) would not occur. We are led to the conclusion that the widths are due to absorption by many $\left(N>10^{2}\right)$ clouds covering the face of the central object and having large relative motions but largely thermal internal motions. (Holt et al. 1980 came to the same conclusion concerning the number of clouds responsible for the X-ray absorption from an argument based on typical cloud sizes and the fact that a line of sight intercepts one or two clouds.)

The location of the absorbing material relative to emitting BLR clouds can be deduced from the absorption line data (see Anderson 1974). If the absorbing clouds are the same as those causing the X-ray extinction, then it seems clear that absorption lines can nearly fully extinguish the continuum over the line width. An open question is the issue of whether absorption lines have also extinguished part of the overlying emission lines (Anderson 1974). The depth of absorption lines in high-resolution spectra with good signal-to-noise ratios (Anderson and Kraft 1969; Anderson 1974) is typically only $20 \%-40 \%$ of the adjacent continuum, and extinction of the continuum alone seems a good possibility. Low-resolution $I U E$ data are less clear on this point (Penston et al. 1979; Penston et al. 1981), and high signal-to-noise and spectral resolution studies of the ultraviolet lines should be pursued when the appropriate instrumentation is available.

The fact that virtually the entire blue half of the Ly $\alpha$ emission line is missing (see Penston et al. 1979) is in agreement with line transfer calculations for outflowing gas (see Ferland and Netzer 1979; Ferland, Netzer, and Shields 1979; Kwan and Krolik 1981). The $\mathrm{H}^{0}$ zone beyond the $\mathrm{H}^{+}$ionization front is highly opaque to Ly $\alpha$ photons, and individual clouds strongly direct Ly $\alpha$ radiation back through the $\mathrm{H}^{+}$zone toward the source of ionizing radiation. Although this affects the observed line profile by permitting only radiation from clouds on the far side of the central object to be visible in Ly $\alpha$, the total power in the line should not be affected if the BLR is roughly spherically symmetric about the source of ionizing radiation, as Holt et al. (1980) argue.
The optical and ultraviolet studies have also clearly shown that the absorption lines vary in both intensity and velocity. Anderson (1974) found that the Balmer lines are much more variable than the adjacent $\mathrm{He}$ I $\lambda 3889$ line and reported that significant changes occur over time scales as short as 30 days. Penston et al. (1981) also found the absorption component of several ultraviolet lines to be variable, but apparently with a longer time scale.

Finally, two apparent conundrums posed by the optical, ultraviolet, and X-ray data deserve mention. First, the large column density of absorbing matter inferred by Holt et al. (1980) would be sufficient to fully extinguish the optical continuum were the gas characterized by a normal (i.e., galactic) dust-to-gas ratio (see Mushotzky, Holt, and Serlemitsos 1978). For comparison, Penston et al. (1981) find a small $E(B-V)$, between 0.05 and $0.10 \mathrm{mag}$. A second problem lies in reconciling the column densities inferred by different studies. The X-ray data typically require $N \sim 10^{22}-10^{23} \mathrm{~cm}^{-2}$, while the ultraviolet absorption lines imply a far smaller column, only $N \sim 3 \times 10^{19} \mathrm{~cm}^{-2}$. The resolution of these problems is discussed in $\S \mathrm{IV}$.

\section{COMPARISON WITH MODEL CALCULATIONS}

The evidence outlined above suggests that the line and continuous absorption in NGC 4151 arises in a large number of BLR clouds which lie along the line of sight to the nucleus. In this section we compare predictions of photoionization calculations with the optical and ultraviolet emission line and X-ray continuum data to test the validity of these models.

\section{a) The Emission-Line Spectrum}

Published emission-line data will be used to deduce the physical conditions and characteristics of both BLR and NLR clouds in NGC 4151. Osterbrock and Koski (1976) report moderate resolution relative spectrophotometry covering the ground-based optical spectrum while Penston et al. (1981) report the results of extensive satellite ultraviolet observations. We compile a mean emission-line spectrum by first correcting these intensities for reddening and then normalizing these data to a common intensity scale. The data are summarized in Table 1 where the first column gives the line identification and the second column the observed intensities relative to $\mathrm{He}$ II $\lambda 1640$ for the ultraviolet data, and relative to $H \beta_{n}$, the sharp component of $H \beta$, for the optical data. In the case of the UV data these intensities are the average values for 1978 October and December, and 1979 January. We assume a reddening of $E(B-V)$ $=0.1 \mathrm{mag}$. This is consistent with Penston et al.'s (1981) estimate of $E(B-V)=0.05-0.10 \mathrm{mag}$ from the weakness of the $\lambda 2200 \AA$ feature, and was deduced by Allen's (1979) method and the Lick [O II] and [S II] data.

Unfortunately the UV and optical data cannot be compared directly since the $I U E$ and Lick observations 
TABLE 1

EMISSION-LINE INTENSITIES

\begin{tabular}{|c|c|c|c|c|c|c|}
\hline (1A) & $\begin{array}{l}\text { tion } \\
\text { (1B) }\end{array}$ & $\begin{array}{l}I_{\mathrm{obs}}{ }^{\mathrm{a}} \\
(2)\end{array}$ & $\begin{array}{l}I_{\text {cor }}^{\mathrm{b}} \\
(3)\end{array}$ & $\begin{array}{c}\text { Total } \\
(\mathrm{NLR}+\mathrm{BLR}) \\
(4)\end{array}$ & $\begin{array}{c}\text { BLR } \\
\log U=-0.75 \\
(5)\end{array}$ & $\begin{array}{c}\text { NLR } \\
\log U=-2.5 \\
(6)\end{array}$ \\
\hline $\mathrm{H}_{\mathrm{I}} \ldots \ldots$. & 1216 & $7.4 \pm 1.5$ & $61.5 \pm 10.5$ & 145.56 & 77.19 & 68.37 \\
\hline $\mathrm{N}$ iv]....... & 1486 & $1.0 \pm 0.4$ & $6.6 \pm 2.2$ & 43.2 & 4.32 & 0.09 \\
\hline $\mathrm{C}$ IV $] \ldots \ldots$ & 1549 & $14.7 \pm 3.7$ & $106.3 \pm 24.3$ & 109.29 & 106.18 & 3.29 \\
\hline He II....... & 1640 & $1.00 \pm 0.2$ & $7.2 \pm 1.7$ & 6.61 & 4.18 & 2.43 \\
\hline $\mathrm{O} \mathrm{III}] \ldots \ldots$ & 1666 & $0.7 \pm 0.2$ & $5.0 \pm 1.7$ & 6.65 & 5.80 & 0.85 \\
\hline $\mathrm{N}$ III] $\ldots$. & 1750 & $0.2 \pm 0.1$ & $1.6 \pm 0.8$ & 1.49 & 1.14 & 0.35 \\
\hline $\mathrm{C}$ III] ...... & 1909 & $2.6 \pm 0.3$ & $17.7 \pm 1.7$ & 13.66 & 8.21 & 5.45 \\
\hline $\mathrm{C}$ II] $\ldots \ldots$ & 2326 & $0.5 \pm 0.1$ & $3.3 \pm 0.6$ & 10.15 & 8.89 & 1.26 \\
\hline Mg II .... & 2798 & $2.0 \pm 0.2$ & $13.3 \pm 1.1$ & 9.98 & 9.01 & 0.97 \\
\hline$[\mathrm{O} \mathrm{II}] \ldots \ldots$ & 3727 & $1.94 \pm 0.66$ & $2.12 \pm 0.73$ & 1.86 & $\ldots$ & 1.86 \\
\hline$[\mathrm{Ne}$ III $] \ldots$ & 3869 & $1.37 \pm 0.02$ & $1.51 \pm 0.04$ & 0.74 & $\ldots$ & 0.74 \\
\hline$[\mathrm{S} \mathrm{II}] \ldots \ldots$ & 4071 & $0.33 \pm 0.07$ & $0.36 \pm 0.10$ & 0.22 & $\ldots$ & 0.22 \\
\hline$[\mathrm{O}$ III $] \ldots$ & 4363 & $0.36 \pm 0.03$ & $0.34 \pm 0.04$ & 0.37 & 0.05 & 0.32 \\
\hline \multirow[t]{2}{*}{ He II...... } & $4686 n$ & $0.19 \pm 0.05$ & $0.19 \pm 0.05$ & 0.33 & . & 0.33 \\
\hline & $4686 \mathrm{~b}$ & $0.77 \pm 0.37$ & $0.77 \pm 0.37$ & 0.52 & 0.52 & \\
\hline [Ar IV] & 4730 & $0.08 \pm 0.04$ & $0.08 \pm 0.04$ & 0.08 & $\ldots$ & 0.08 \\
\hline \multirow[t]{2}{*}{$\mathrm{H}$ I ....... } & $4861 \mathrm{ln}$ & 1.00 & 1.00 & 0.83 & $\ldots$ & 0.83 \\
\hline & $4861 \mathrm{~b}$ & $5.61 \pm 1.03$ & $5.61 \pm 1.03$ & 5.46 & 5.46 & \\
\hline [O III] & 5007 & $14.46 \pm 1.17$ & $14.41 \pm 1.15$ & 14.41 & $\ldots$ & 14.41 \\
\hline$\left[\mathrm{N}_{\mathrm{I}}\right] \ldots$ & 5199 & $0.10 \pm 0.01$ & $0.10 \pm 0.01$ & 0.04 & $\ldots$ & 0.04 \\
\hline \multirow[t]{2}{*}{ He I } & $5876 n$ & $0.19 \pm 0.05$ & $0.17 \pm 0.05$ & 0.12 & & 0.12 \\
\hline & $5876 \mathrm{~b}$ & $1.29 \pm 0.51$ & $1.15 \pm 0.47$ & 0.58 & 0.58 & \\
\hline$[\mathrm{O} \mathrm{I}]$ & 6300 & $0.85 \pm 0.13$ & $0.76 \pm 0.12$ & 0.76 & $\ldots$ & 0.76 \\
\hline$[\mathrm{Fe} \mathrm{x}] \ldots$ & 6374 & 0.08 & 0.07 & 0.09 & 0.07 & 0.02 \\
\hline \multirow[t]{2}{*}{$\mathrm{H}$ I ........ } & $6563 n$ & $4.47 \pm 1.17$ & $3.93 \pm 1.03$ & 3.38 & $\ldots$ & 3.38 \\
\hline & $6563 \mathrm{~b}$ & $19.50 \pm 5.09$ & $17.13 \pm 4.90$ & 21.23 & 21.23 & \\
\hline$[\mathrm{N}$ II $] \ldots . .$. & 6583 & $2.21 \pm 0.03$ & $2.13 \pm 0.03$ & 1.23 & $\ldots$ & 1.23 \\
\hline
\end{tabular}

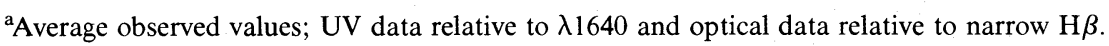

are not simultaneous, and the Lick data do not have an absolute calibration. The optical and ultraviolet data can be brought to a common intensity scale by normalizing the $\mathrm{He}$ II lines to $1640 / 4686=7.5$ (Seaton 1978). Unlike hydrogen lines, intensities of $\mathrm{He}$ II lines should not be affected by resonance line trapping since $\mathrm{He}$ II Ly $\alpha$ is quickly destroyed by both photoionization of $\mathrm{H}^{0}$ and the Bowen fluorescence mechanism (see Weymann and Williams 1969; see also MacAlpine 1981). This renormalization largely removes any uncertainties introduced by the reddening correction.

Several additional sources of uncertainty remain. In general, individual lines will contain contributions from both BLR and NLR clouds. The Lick data have sufficient resolution to devolve the two components, but the $I U E$ spectra do not. Further, the continuum is variable, often by a factor of 2, and BLR line intensities could follow this variation (see Boksenberg and Netzer 1977). The NLR clouds are more distant and are expected to remain constant from light travel time arguments. This type of argument led Penston et al. (1981) to suggest that C IV and Ly $\alpha$ were largely formed in the BLR while $\mathrm{C}$ III] and $\mathrm{Mg}$ II have a significant contribution from NLR gas. Another source of concern is presented by the absorption components which may affect the intensities of some lines (e.g., C IV) by as much as $\sim 30 \%$. The error estimates in columns (2) and (3) of Table 1 reflect only the internal error in repeated measurements of line intensities and do not include systematic errors introduced by these effects.

\section{b) Model Calculations - BLR Clouds}

Photoionization models are characterized by (see Davidson and Netzer 1979) (1) the shape and luminosity of the ionizing continuum; (2) the density and chemical composition of the clouds; (3) the distance between the central engine and the clouds, and finally, in the case of photoionization by a hard X-ray continuum, (4) the thickness of the clouds. The latter follows because hard radiation easily penetrates the clouds to great depths, creating an extensive warm, neutral zone behind the classical $\mathrm{H}^{+}-\mathrm{H}^{0}$ Strömgren sphere (see Weisheit, Shields, and Tarter 1981). In previous calculations (cf. Kwan and Krolik 1981) the depth of this neutral zone was set by truncating the clouds when weak lines which originate in this zone (such as C II] $\lambda 2326$ ) reached observable strengths. 
NGC 4151 is a good object for study because many parameters affecting model calculations have been set by other considerations. Figure 1 shows a schematic illustration of the assumed continuum. This continuum is in general agreement with observations, although we make no attempt to reproduce weak additional components (see Penston et al. 1981). We assume that the unobserved extreme ultraviolet $(13.6<h \nu<500 \mathrm{eV})$ continuum has also been extinguished by the same leaky absorber as the soft X-rays. In our calculations we will assume that the $\mathrm{X}$-ray low-frequency cutoff originates in BLR gas and that the continuum incident upon the inner face of these clouds is given by extrapolating both the observed ultraviolet and X-ray continua to intermediate frequencies. We also assume that the absorption in each line of sight occurs in a single cloud and will use this extinction to set the thickness of the cloud. Radial integrations are stopped when the photon densities, per unit energy interval, have fallen to $N(2 \mathrm{keV}) / N(3.5$ $\mathrm{keV})=0.3$. This corresponds to a column density of $N_{\mathrm{H}}=10^{22.9} \mathrm{~cm}^{-2}$ for a continuum with a spectral energy slope of $\alpha=-0.5$ (see Cruddace et al. 1974). This column density is a value intermediate between the extremes exhibited by NGC 4151 (Barr et al. 1977) and should be fairly characteristic.

Holt et al. (1980) have argued that there are typically two clouds per line of sight, so the second cloud lies in the shadow of the first. The ionization structure of this ensemble should be largely the same as that of a thicker single cloud. Line transfer effects, which are very important in BLR clouds, would of course be mitigated if the pair of clouds have relative velocities which are large

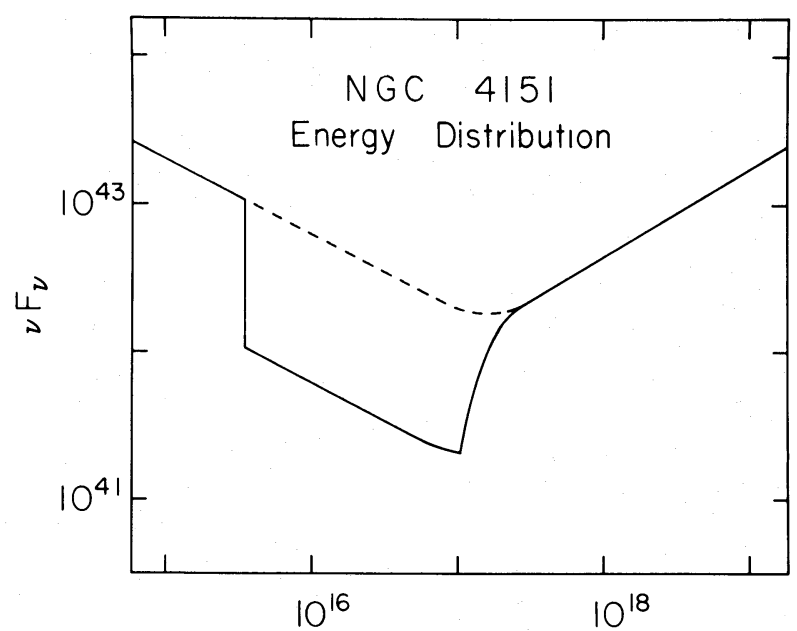

FIG. 1.- Ionizing Continuum. The observed and inferred ionizing continuum of NGC 4151 is shown. A distance of $20 \mathrm{Mpc}$ is assumed. The observed continuum has been corrected for this distance and is plotted as ergs $\mathrm{s}^{-1}$. Low frequencies are dominated by a steep falloff $\left(f_{\nu} \propto \nu^{-1.5}\right)$, while the X-ray and $\gamma$-ray continuum has a more gentle slope $\left(f_{\nu} \propto \nu^{-0.5}\right)$. The continuum with $\nu>1$ ryd is assumed to be extinguished by a leaky absorber covering $90 \%$ of the source as described by Holt et al. 1980 . compared to the thermal line width or if the clouds are widely separated.

Since it is our intention to see whether the lowfrequency absorption can be understood in the context of "classical" BLR models, we employ standard assumptions concerning density and chemical composition and do not vary these parameters. A solar chemical composition will be assumed for the eleven elements we treat (see Lambert 1978; Lambert and Luck 1978; we assume $\mathrm{He} / \mathrm{H}=0.1$ ). There is some disagreement as to whether heavy elements are enriched (Davidson and Netzer 1979) or depleted (Kwan and Krolik 1981) in quasar BLR clouds, but a roughly solar iron abundance seems to be indicated by the X-ray Fe $K$-edge absorption feature (see Mushotzky, Holt, and Serlemitsos 1978).

A constant density of $N=10^{9.5} \mathrm{~cm}^{-3}$ will be assumed. Constant pressure models are probably more appropriate (see Baldwin and Netzer 1978), but constant density models allow a more meaningful comparison between clouds with different ionization parameters. Tests show that the differences between the two types of models are comparable to the uncertainties in other aspects of the calculations (i.e., the atomic data base). The dimensionless ionization parameter used here is $U=Q(\mathrm{H}) / 4 \pi r^{2} c N_{e}$ and is the ratio of ionizing photons to free electrons, while the surface density of ionizing photons at the inner face of the cloud is $\phi_{\mathrm{H}}=$ $Q(\mathrm{H}) / 4 \pi r^{2}$. In these expressions $Q(\mathrm{H})$ is the number of ionizing photons emitted by the central object per unit time (see Osterbrock 1974), $r$ is the separation between the central engine and the clouds, and $c$ and $N_{e}$ are the speed of light and electron density, respectively.

Calculations are performed with the program described by Ferland and Truran (1981). The basic assumptions and techniques are similar to those outlined by Netzer (1980), Weisheit, Shields, and Tarter (1981), and Kwan and Krolik (1981). Radiative and collisional processes involving hydrogen, which we model as a five-level atom, are treated as described by Netzer (1980) and Kwan and Krolik (1981) with rates taken from Kwan and Krolik (1981) and Drake and Ulrich (1980). Line trapping is treated in the mean escape probability formalism (see Capriotti 1965) with escape probabilities taken from Adams (1972) for resonance lines and Canfield and Puetter (1981) for subordinate transitions. Diffuse fields produced by recombining hydrogen and helium are treated by $\Lambda$-iteration, as in Williams (1967).

Test runs show general agreement between these calculations and published models. Discrepancies which do exist can largely be traced to some physical processes which we treat but which are not included in some other calculations. For example, our continuum extends to $100 \mathrm{keV}$ and we find Compton recoil ionization of $\mathrm{H}^{0}$ by $h \nu>2.3 \mathrm{keV}$ radiation in the largely neutral gas beyond the $\mathrm{H}^{+}$Strömgren sphere to be a significant heating and ionization mechanism. Heating and ionization efficiencies for suprathermal electrons in neutral 


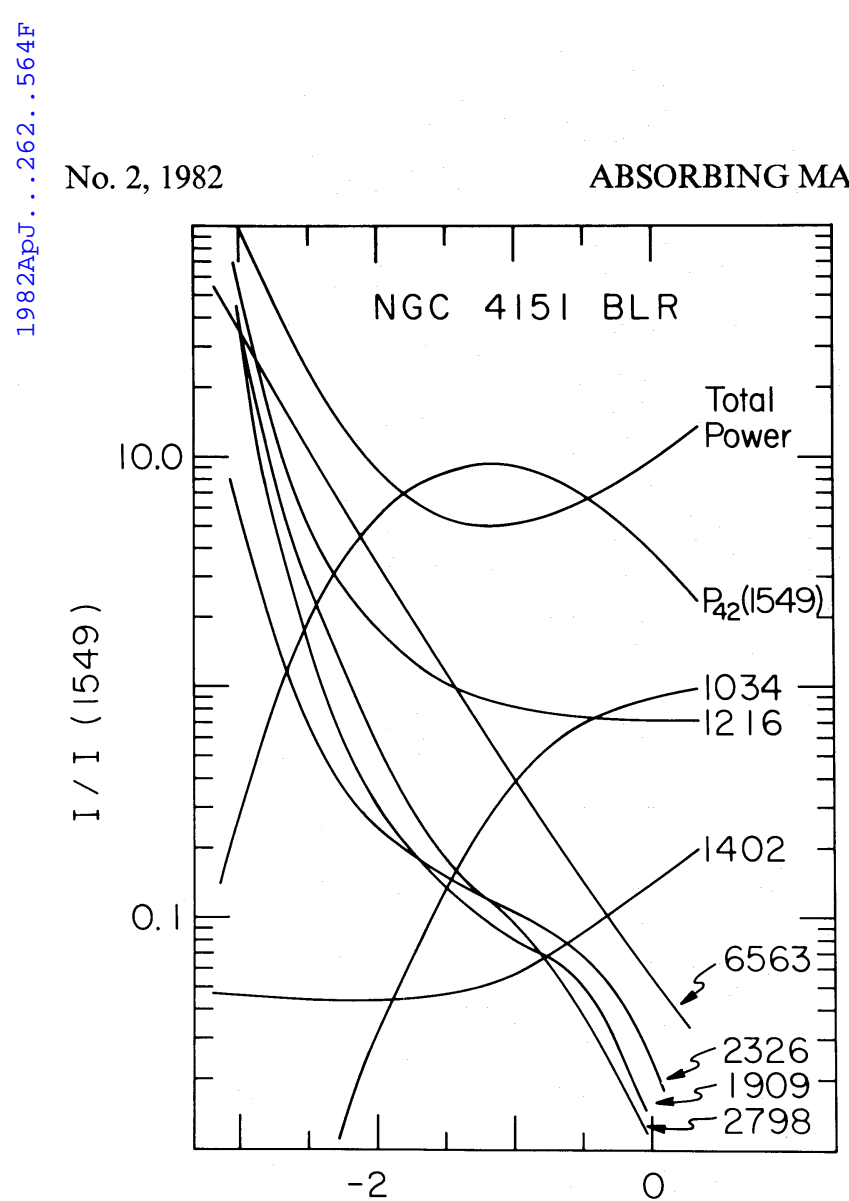

LOG (U)

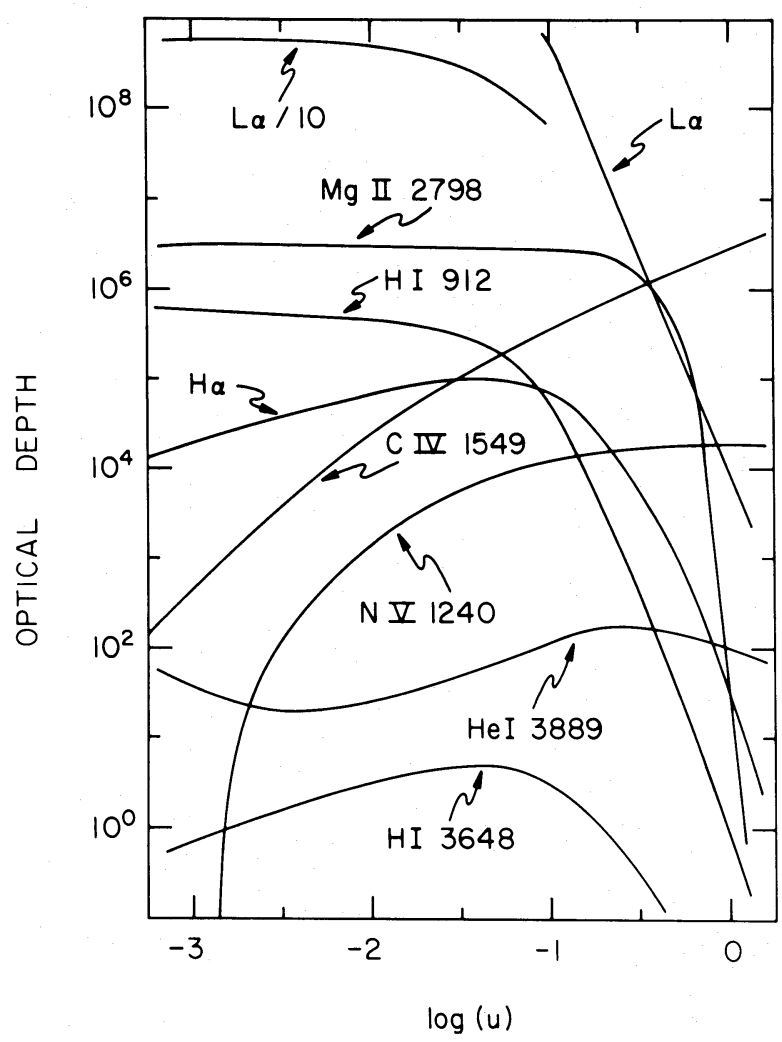

FIG. 3

FIG. 2.-Broad Line Region Emission Spectra. The intensities of O vi $\lambda 1034$, Ly $\alpha$, O IV] $\lambda 1402, \mathrm{H} \alpha, \mathrm{C}$ II] $\lambda 2326, \mathrm{C}$ III] $\lambda 1909$, and Mg II $\lambda 2798$, relative to C IV $\lambda 1549$ are shown as a function of the ionization parameter. The models have a constant hydrogen density of $N=3 \times 10^{9} \mathrm{~cm}^{-3}$, a solar chemical composition, and an ionization parameter $U$ defined as the ratio of ionizing photons to free electrons at the inner face of the cloud. Radial integrations in all models were stopped when the soft X-ray continuum had been extinguished to a relative photon density of $N(2 \mathrm{kev}) / N(3.5 \mathrm{kev})=0.3$. The top line shows the total power radiated by all cooling, recombination, and continuum processes relative to the power radiated in C IV $\lambda 1549$. Over a wide range of the ionization parameter the C iv line carries between $10 \%$ and $20 \%$ of the total power radiated and can be used to reliably estimate the total energy budget of the system. The total power radiated by $\mathrm{C}$ IV $\lambda 1549$, assuming full coverage, is shown in units of $10^{42} \mathrm{ergs} \mathrm{s}^{-1}$. A comparison between available and observed fluxes suggests essentially $100 \%$ coverage of the continuum source by BLR clouds.

Fig. 3.- Line and Continuum Optical Depths. The optical depths at line center for several of the important emission lines are shown as a function of the ionization parameter. Thermal broadening alone is assumed. Optical depths at the hydrogen ionization edges for both the ground and first excited states are also shown.

gas are taken from the Monte Carlo simulations of Shull (1979). Both three-body and dielectronic recombination compete with radiative recombination in some of these models. Three-body rates are taken from Burgess and Summers (1976) and typically increase the total recombination rate by $\sim 20 \%-50 \%$ for singly ionized species. The dielectronic recombination rates of Summers (1974) have been supplemented with Storey's (1981) calculations for nebular temperatures. Storey's rates pertain in the low density limit and have been scaled to account for collisional ionization before stabilization as described by Davidson (1975).

The results of a large number of calculations are shown in Figures 2 and 3. Only the ionization parameter has been allowed to vary, which is equivalent to changing the distance between the clouds and the source.
Figure 2 shows the intensities of several of the strong cooling lines relative to $C$ iv $\lambda 1549$, and Figure 3 shows optical depths of several lines and continuum edges, integrated through the cloud. Line optical depths assume thermal broadening alone.

In the case of BLR clouds in high redshift QSOs the ionization parameter is usually determined by matching the ionization sensitive ratio (C III] $\lambda 1909$ /C IV $\lambda 1549$ ) (see Davidson 1977; Davidson and Netzer 1979). This line ratio is typically $\sim 0.5$ (Baldwin 1977a) for high redshift QSOs and corresponds to an ionization parameter $U \approx 10^{-2.5}$ for these objects. The ionization parameter for BLR clouds in NGC 4151 must be considerably larger than this because of the relative weakness of the $\lambda 1909$ line in this object. Penston et al. (1981) use variability arguments to show that most C IV emission 
originates in BLR gas while a significant part of the C III] line must originate in NLR gas. This suggests that In the intrinsic BLR $1909 / 1549$ ratio is considerably - smaller than the observed value of 0.17 . This in turn requires an ionization parameter greater than $U \approx 10^{-1.5}$.

Figure 2 shows that the ratio $I(\mathrm{H} \alpha) / I(\mathrm{C}$ IV $)$ is a sharp function of $U$. This is because the grid of models has a total column density which is nearly constant, while models with larger $U$ have a larger $\mathrm{H}^{+}$zone (where all $\mathrm{C}$ IV emission is formed) and a correspondingly smaller $\mathrm{H}^{0}$ zone (where essentially all the Balmer emission is formed). The observed ratio of the broad component of $\mathrm{H} \alpha$ to the total $\mathrm{C}$ IV is $I(6563) / I(1549)=$ $0.16 \pm 0.05$ and corresponds to $U \approx 10^{-0.75}$. We note that in the case of the average QSO (Baldwin 1977a; Davidson and Netzer 1979) this ratio is $\mathrm{H} \alpha / \mathrm{C}$ IV $\approx 3$, corresponding to $U \approx 10^{-2}$, in agreement with the ionization parameter deduced from the $\mathrm{C}$ III]/C IV ratio.

The Balmer continuum optical depth predicted by these models also suggests a large ionization parameter (see Fig. 3). The near-ultraviolet spectrum of NGC 4151 shows no absorption at the Balmer jump (see Boksenberg and Penston 1976), while models with $U<$ $10^{-1}$ are optically thick to $\lambda 3648 \AA$ radiation (see also Kwan and Krolik 1981). Thus two independent lines of evidence, emission from the entire ensemble of BLR clouds and the absence of an absorption Balmer jump from line-of-sight clouds, both demand an uncharacteristically large ionization parameter. Both the reason for the large ionization parameter in this low-luminosity Seyfert, as well as the reason that high redshift QSOs tend to favor the same lower ionization parameter, are presently unclear (see Davidson 1977).

Figure 4 shows some details of the structure of the model with $U=10^{-0.75}$. The upper part of the figure shows the fractional ionization of hydrogen and helium. The hydrogen ionization at depths $\delta r>10^{13} \mathrm{~cm}$ is maintained by photo- and collisional ionization from excited states, mainly $n=2$ and $n=3$. Because of the large ionization parameter, this residual ionization is much larger than is typically found (see Kwan and Krolik 1981; Weisheit, Shields, and Tarter 1981). The lower part of the figure shows the electron temperature and the radiative acceleration.

The run of the radiative acceleration, $g_{\text {rad }}$, with depth in this model has an interesting implication. Since $g_{\text {rad }}$ is mainly due to photoionization of hydrogen throughout this model, the acceleration can be expressed as

$$
g_{\mathrm{rad}}=\frac{h \bar{\nu}}{C} \frac{\alpha N_{e}}{\mu} \approx 0.35\left(\frac{h \bar{\nu}}{10 \mathrm{eV}}\right) \mathrm{cm} \mathrm{s}^{-2},
$$

because of the balance between photoionization and recombination (see Blumenthal and Mathews 1975). In this expression $\alpha=4 \times 10^{-13} \mathrm{~cm}^{3} \mathrm{~s}^{-1}$ is the $\mathrm{H}^{+}$recombination coefficient and $\mu=2.3 \times 10^{-24} \mathrm{~g}$ is the mean mass per proton. For $\delta r<10^{13} \mathrm{~cm}$ the hydrogen ioniza-

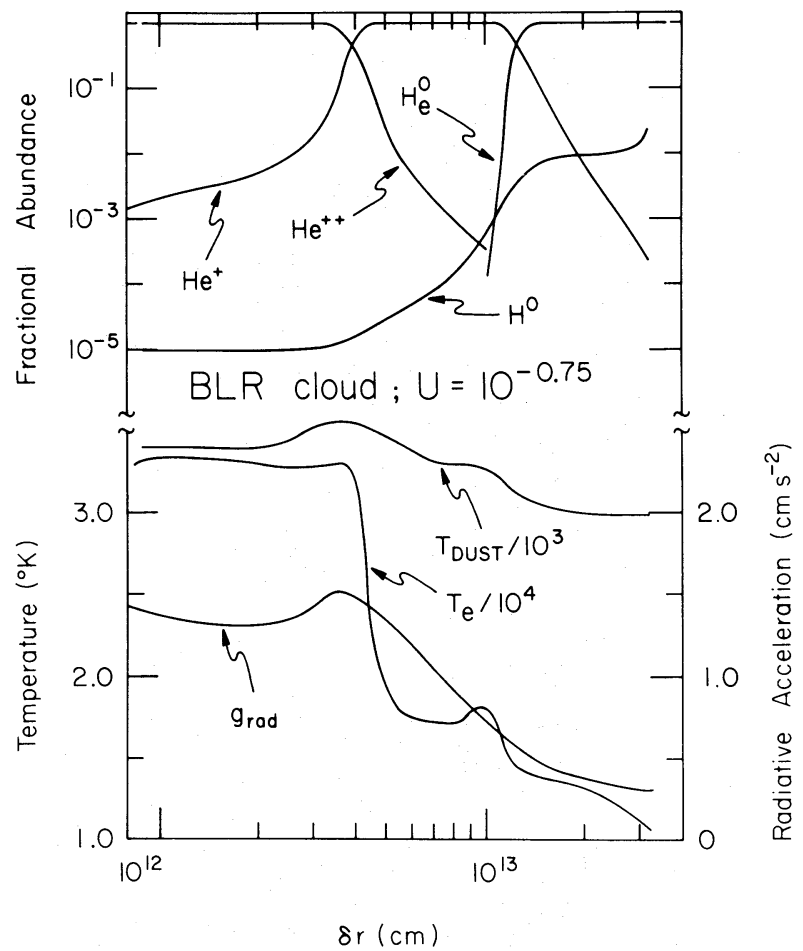

FIG. 4.-Broad Line Region Cloud Ionization Structure. The ionization and thermal structure of the best fitting BLR model is shown. The cloud has an ionization parameter of $U=10^{-0.75}$ and a total thickness of $3.1 \times 10^{13} \mathrm{~cm}$, as deduced from the X-ray absorption. The radius is given as the depth from the inner face of the cloud, and the fractional ionization of hydrogen and helium is shown at the top. The hydrogen ionization for $\delta r>1.1 \times 10^{13} \mathrm{~cm}$ is maintained largely by photoionization from excited states. The electron temperature as well as that of hypothetical graphite grains of radius $0.05 \mu \mathrm{m}$ is also shown, together with the radiative acceleration produced by both line and continuum absorption. Graphite grains would quickly sublimate in this environment as a result of the large ionization parameter. The radiative acceleration is fairly constant across the cloud because hydrogen has remained ionized.

tion is maintained mainly by ground-state absorption of relatively hard radiation $(h \nu \sim 40 \mathrm{eV})$ and $g_{\text {rad }} \approx 1.4$ $\mathrm{cm} \mathrm{s}^{-2}$. In deeper layers $\left(\delta r>10^{13} \mathrm{~cm}\right)$ Balmer continuum absorption of $h \nu \approx 10 \mathrm{eV}$ radiation is the dominant ionization mechanism and $g_{\text {rad }} \approx 0.4 \mathrm{~cm} \mathrm{~s}^{-2}$. The important point is that, because hydrogen remains ionized throughout, $g_{\text {rad }}$ is fairly uniform across the model and does not show the dramatic decline at the $\mathrm{H}^{+}-\mathrm{H}^{0}$ ionization front which occurs when this front is present (see Blumenthal and Mathews 1975, 1979; Netzer 1980). This uniformity makes coherent acceleration possible without the serious Rayleigh-Taylor instability which normally plagues such models. Both this result and the absorption line data outlined above strongly support the idea that NGC 4151 is losing mass in a radiatively accelerated outflow. We note in passing that the radial thickness of the clouds deduced here, $\delta r \approx$ $10^{13.5} \mathrm{~cm}$, is of order the projected size of $\delta r \approx 10^{13} \mathrm{~cm}$ 
inferred by Holt et al. (1980). Apparently the shape of BLR clouds is more similar to that of biscuits or dumplings rather than to pancakes. These results strongly support the general picture of line formation in a radiatively driven outflow as developed by Blumenthal and Mathews (1975).

Figure 4 also shows the equilibrium temperature of hypothetical graphite grains with a radius of $0.03 \mu \mathrm{m}$. This temperature was computed as in Martin and Ferland (1980) assuming their opacity function and equating heating and cooling processes as described by Clayton and Wickramasinghe (1976). Graphite grains, which are among the most resilient with a sublimation temperature of $\approx 2000 \mathrm{~K}$, cannot survive in this environment. (Graphite could survive were the ionization parameter given the conventional lower value, as was shown by Shuder and MacAlpine 1979 and by Martin and Ferland 1980). The fact that grains would be quickly destroyed resolves the paradox between the large reddening predicted from X-ray column densities (Mushotzky, Holt, and Serlemitsos 1978) and the small extinction actually observed (Penston et al. 1981).

Column (5) of Table 1 summarizes predicted line intensities for the model with $U=10^{-0.75}$. Predicted values are normalized to the observed intensity of the $\mathrm{C}$ IV line. One important prediction concerning the covering factor of BLR clouds will be mentioned before proceeding. As Figure 2 shows, the $C$ IV $\lambda 1549$ line represents a significant fraction $(\approx 10 \%-20 \%)$ of the total power radiated by the clouds over a wide range of the ionization parameter. It is because the $\mathrm{C}$ iv line is a major coolant that its intensity is especially reliably predicted (see Davidson 1973). This follows because the line intensity is coupled to the total heating rate rather than to details of the model, such as temperature or ionization fraction, which strongly affect intensities of weak lines. A comparison between observed and predicted $\mathrm{C}$ iv fluxes should provide a reliable estimate of the covering factor. Figure 2 shows that the total power radiated by $\lambda 1549$ is predicted to be $L(1549)=8 \times 10^{42}$ ergs $\mathrm{s}^{-1}$ assuming full coverage. Since the reddening corrected C IV power is $10^{42.6 \pm 0.3} \mathrm{ergs} \mathrm{s}^{-1}$ (Penston et al. 1981), the energetics alone demand essentially full coverage of the continuum source by BLR clouds $(\varepsilon \approx$ $0.5_{-0.25}^{+0.5}$ ). Since a very large covering factor for low luminosity Seyferts has already been deduced from X-ray observations (Mushotzky 1982), this logic can be reversed to argue that photoionization is the dominant heating and ionization source of BLR clouds. Photoionization has always been a simple but ad hoc assumption, and the role of other sources of heating such as shocks due to cloud-cloud collisions (see the discussion by Daltabuit, MacAlpine, and Cox 1978) has always been unclear. The example of NGC 4151 shows that additional sources of energy to the BLR must be small compared to the ionizing continuum alone in at least some Seyfert galaxies.
Both free-free and free-bound radiative interactions in the BLR gas are expected to contribute part of the observed continuum (see Weisheit, Shields, and Tarter 1981; Kwan and Krolik 1981). Our calculations predict that $\approx 10 \%$ of the continuum near $\mathrm{H} \beta$ is produced by bremsstrahlung emission. Together bremsstrahlung and Balmer recombination account for $\approx 30 \%$ of the $\lambda 3000$ $\AA$ continuum, but these processes make a negligible contribution to the continuum shortward of $\lambda 2000 \AA$ and cannot account for the $\lambda 1455 \AA$ excess discussed by Penston et al. (1981). Emission from the shell should be included in any modelling of the UV-optical continuum, however (see also Malkan and Sargent 1982).

\section{c) Model Calculations - NLR Clouds}

Having established that BLR material covers essentially the entire source of ionizing photons, we now examine the consequences of the absorption of $h \nu<3$ $\mathrm{keV}$ radiation on the physical conditions in the more distant NLR clouds. This analysis is also necessary because emission from NLR clouds can affect intensities of several of the diagnostic emission lines used above.

A constant density of $N_{\mathrm{H}}=10^{3.5} \mathrm{~cm}^{-3}$, as deduced by Allen's (1978) method and the [O II] and [S II] lines, will be assumed. The results are not sensitive to this density over a range of about a factor of 10 because of the homology relation introduced by the ionization parameter. The ionizing continuum shown by the solid line in Figure 1, which assumes $90 \%$ coverage of the continuum source by BLR gas, is assumed to ionize the NLR clouds. This continuum is the only major difference between these calculations and those presented by Ferland (1981). As in the case of the BLR clouds, hard $\mathrm{X}$-rays penetrate to great depths and a stopping criterion is needed to set the thickness of the clouds. Tests show that too thick a cloud will produce exceptionally strong [O I], [O II], and [S II] lines. These lines arise in the warm, largely suprathermal electron heated and ionized $\mathrm{H}^{0}$ zone and can give the spectrum an appearance similar to that of a shock heated galaxy such as NGC 1052 (Fosbury et al. 1978; Ferland and Netzer 1982). In these models the strength of $\left[\begin{array}{ll}O_{I} & \lambda 6300\end{array}\right.$ relative to the sharp component of $\mathrm{H} \beta$, a line ratio which is quite sensitive to the total thickness of the cloud, is used to truncate the thickness of the clouds.

Figure 5 shows the results of these calculations assuming a solar mixture of heavy elements. Nowhere are the predictions of the $[\mathrm{O} \mathrm{III}]$ and $[\mathrm{O} \mathrm{II}]$ lines in agreement with the observed values. The temperature-density sensitive ratio [O III] $4363 / 5007$ is always $\sim 3$ times too small, which suggests that the models have either too small a temperature or too small a density. The strong [O II] and [S II] lines rule out a much higher density (for a one-component NLR), so apparently the models have underestimated $T_{e}$. A second indication that these models lack an essential ingredient is the $5007 / \mathrm{H} \beta$ ratio, 


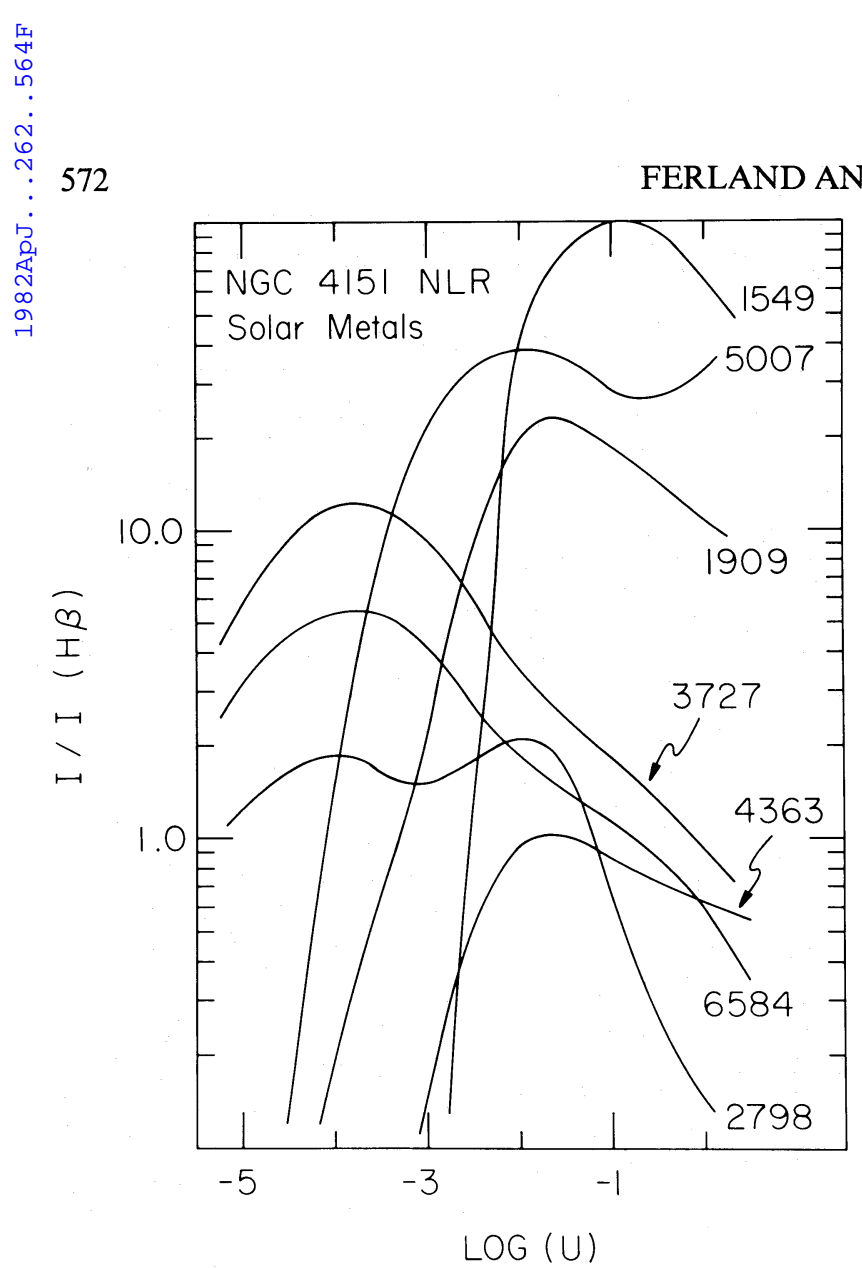

FIG. 5

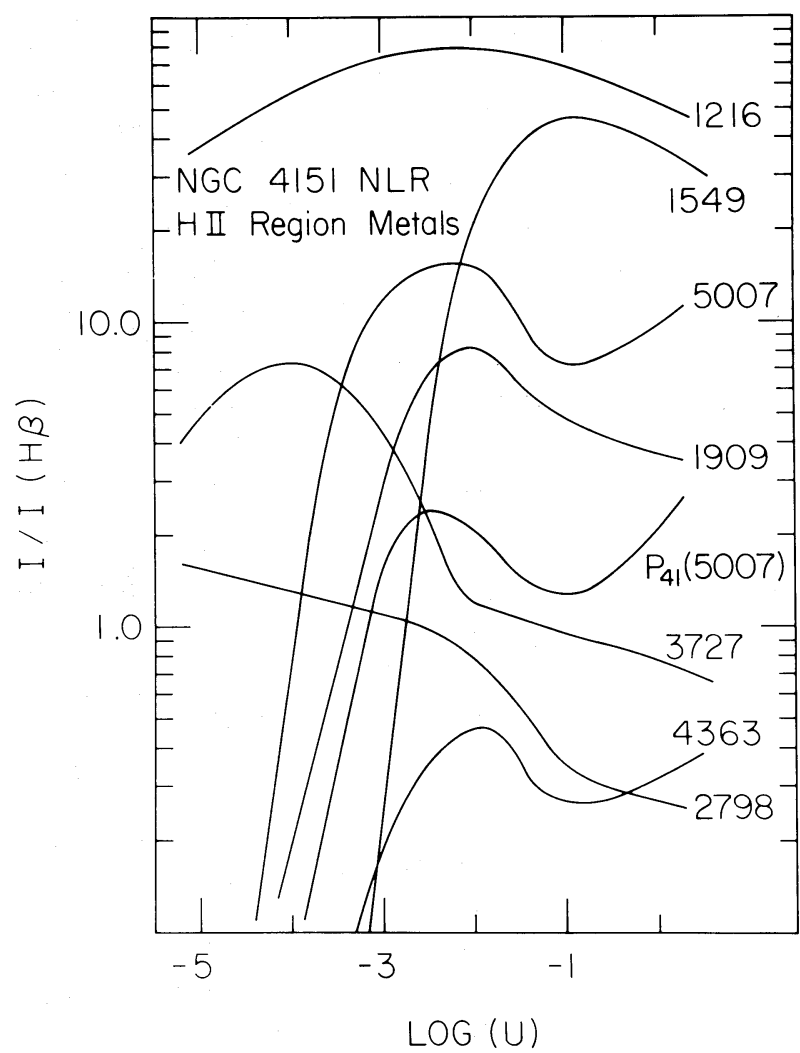

FIG. 6

FIG. 5. - Narrow Line Region Emission Spectra - Solar Abundances. The extinguished continuum shown in Fig. 1 was assumed to ionize a solar abundance gas with a density $N_{\mathrm{H}}=10^{3.5} \mathrm{~cm}^{-3}$ as deduced by Allen's 1979 method and the [S II] and [O II] lines. Intensities of C IV $\lambda 1549$, [O III] $\lambda 5007, \mathrm{C}$ III] $\lambda 1909$, [O II] $\lambda 3727$, [O III] $\lambda 4363, \mathrm{H} \alpha$, and $\mathrm{Mg}$ II $\lambda 2798$, are shown relative to the sharp component of $\mathrm{H} \beta$. Both the larger than observed $5007 / \mathrm{H} \beta$ ratio and the too small $4363 / 5007$ ratio suggest that the heavy elements are depleted in the NLR clouds.

FIG. 6. - NLR Spectra-H II Region Abundances. These models are identical to those of Fig. 4 with the exception that all metals are assumed to be depleted by a factor of 3 relative to a solar mixture. This composition is similar to those of extragalactic giant $\mathrm{H}$ II regions (see French 1980). The total power radiated by $\mathrm{O}$ III $\lambda 5007$, in units of $10^{41} \mathrm{ergs} \mathrm{s}^{-1}$ and assuming full coverage of the continuum source by NLR clouds, is also shown.

which is a factor of $\sim 3$ too large for most values of $U$. This ratio is sensitive to both the shape of the ionizing continuum and the oxygen abundance. The dependence on the shape of the continuum is caused by the fact that the [O III] lines are among the strongest coolants in the gas, so their strength is proportional to the total heating rate. NLR Balmer lines are largely formed by recombination, and their intensities are in turn related to the photoionization rate. The line ratio is thus a measure of the mean energy per ionizing photon (see also Kaler 1978). The differences between Figure 5 and the results of Ferland (1981) are largely due to the fact that the ionizing continuum in the present calculations is dominated by relatively hard radiation because of the absorption by BLR gas.

Both the too large [O $\mathrm{III}] \lambda 5007 / \mathrm{H} \beta$ ratio and the too small $4363 / 5007$ ratio can be corrected by lowering the abundances of the heavy elements. This is because the electron temperature rises to maintain the same total cooling rate as the metallicity is decreased (see Osterbrock 1974). Figure 6 shows the results of calculations with the abundances of all metals reduced by a factor of 3 relative to hydrogen. This depletion is typical of metallicities encountered in extragalactic giant $\mathrm{H}$ II regions (French 1980). For simplicity we consider depletion of all heavy elements by the same factor, although the results are mainly sensitive to the oxygen abundance because of its importance as a gas coolant. Several line ratios are in good agreement with observed values over a wide range of $U$. A value of $U=10^{-2.5}$ is obtained by simultaneously matching the temperature sensitive $4363 / 5007$ ratio, the abundance sensitive $5007 / \mathrm{H} \beta$ ratio, and the ionization sensitive $3727 / 5007$ ratio.

The results of these calculations are given in column (6) of Table 1. Predicted values are normalized to the observed intensity of $[\mathrm{O} \mathrm{III]} \lambda 5007$. As in the case of the BLR, a comparison between observed and predicted fluxes suggests full coverage of the source by NLR gas. 
Figure 6 shows the total power radiated by [O III] $\lambda 5007$, in units of $10^{41} \mathrm{ergs} \mathrm{s}^{-1}$ and assuming full coverage. At the deduced value of the ionization parameter the predicted power is $P(5007)=10^{41.3}$ ergs $\mathrm{s}^{-1}$. Scaling the observed power in the $\mathrm{C}$ IV line by the mean spectrum of Table 1 we find a 5007 flux of $10^{41.6 \pm 0.3}$ ergs $\mathrm{s}^{-1}$. This demands essentially full coverage, or $\varepsilon \approx 0.50_{-0.25}^{+0.5}$. The total emission (the sum of both NLR and BLR contributions) for each line is given in column (4) of Table 1.

One interesting prediction of the NLR calculations concerns the hydrogen lines. For the case of solar abundances the hydrogen spectrum is close to simple case B predictions. This is not true for the case of depleted metals, however. Here, the electron temperature is high enough for collisional excitation of both Lyman and Balmer lines to be important; both $\mathrm{Ly} \alpha / \mathrm{H} \beta$ and $\mathrm{H} \alpha / \mathrm{H} \beta$ are larger than their values for pure recombination. This prediction seems to be consistent with the Lick NLR observations which show a steep Balmer decrement even after correcting for the reddening inferred from the optical spectrum. The possible importance of collisional excitation of hydrogen lines from the ground state has already been mentioned by Netzer (1982) for a denser NLR gas, and by Netzer and Davidson (1979) for BLR gas and a continuum similar to the one we have adopted here. This confirms Netzer's (1982) result that the narrow hydrogen lines are not reliable reddening indicators.

\section{d) Infrared Emission and Discrepant Lines}

With a few exceptions, the predicted composite spectrum is in satisfactory agreement with observations. The most serious discrepancy is in the predicted strength of Ly $\alpha$, a factor of $\approx 2.5$ too strong. This difference could be caused by either one of two effects. The Ly $\alpha$ line is highly asymmetric (Penston et al. 1979), presumably as a result of inward beaming of resonance line radiation in an outflowing gas. The net flux in the line will be unaffected by this beaming only if the emission line region is symmetric about the central object. A breakdown in this symmetry could weaken Ly $\alpha$ relative to lines which are radiated more isotropically (e.g., C IV, C III]) if more clouds approach on the near side of the central object than recede on the far side. A second and more likely possibility is that part of Ly $\alpha$ has been destroyed by dust mixed with NLR gas. This seems likely since even the modest reddening of $E(B-V)=0.1$ mag inferred from the [O II] and [S II] lines would be sufficient to destroy $80 \%$ of the Ly $\alpha$ photons created in NLR gas (Ferland and Netzer 1979). Such a destruction of NLR Ly $\alpha$ alone would be sufficient to bring the predicted total Ly $\alpha$ flux to within $3 \sigma$ of the measured flux. This agreement, together with the apparent depletion of the heavy elements deduced above, which is consistent with the needed reddening (Savage and Bohlin
1979), would seem to be a strong argument in favor of the existence of dust mixed with NLR gas. This conclusion confirms the picture outlined by Heckman et al. (1981) in which forbidden line asymmetries are caused by embedded dust. Reradiation from this amount of embedded dust would not be enough to account for the infrared flux observed by Cutri et al. (1981), however. Both the fact that the infrared emission carries a power which is comparable to the total power radiated in the ultraviolet-soft X-ray continuum, and the low temperature $(T \sim 70 \mathrm{~K})$ of dust at the NLR radius suggest that the warm $(T \sim 900 \mathrm{~K})$ luminous component which dominates $\lambda 4 \mu \mathrm{m}$ emission does not originate in NLR material. These constraints, together with the facts that dust cannot survive in the BLR environment (see above) and is observed to be absent (see Mushotzky, Holt, and Serlemitsos 1978) suggest that the infrared excess is not the result of reemission of energy absorbed from the high-energy power-law continuum.

Some minor discrepancies also exist in predicted strengths of [Ne III] and [N II] lines. These could be corrected by smaller depletion factors for nitrogen and neon than for oxygen. Such changes would be in keeping with the analogy to $\mathrm{H}$ II region abundances (see French 1980), but we see little point in "fine-tuning" the models further.

The only other serious difference between predicted and observed values is the $\mathrm{C}$ II] $\lambda 2326$ line. This line is formed in the extended warm $\mathrm{H}^{0}$ zone beyond the $\mathrm{H}^{+}$ Strömgren sphere (see Kwan and Krolik 1981). Several other lines (e.g., the Balmer lines, $\mathrm{Mg}$ II $\lambda 2798$, and many $\mathrm{Fe}$ II lines) compete with $\lambda 2326$ in cooling this region. A likely source of error in our predictions of the $\mathrm{C}$ II] intensity lies with the Fe II lines, which we treat as in Netzer (1980). The thermal equilibrium is especially unreliable in this neutral zone because the atomic data for $\mathrm{Fe}^{+}$is quite uncertain and because many more $\mathrm{Fe}$ II lines are actually observed than are included in the present calculations (see Wills, Netzer, and Wills 1980). NGC 4151 is, of course, a strong source of $\mathrm{Fe}$ II emission (see Netzer 1974). The strength of the C II] line would also be among the most affected by breaking the BLR cloud in half, as suggested by Holt et al. (1980), since the line is strong largely because trapped Ly $\alpha$ and Balmer lines are inefficient coolants.

\section{IMPLICATIONS OF THE MODELS}

\section{a) Size and Kinematics}

The radii of both the BLR and NLR can be estimated since the luminosity of NGC 4151 in ionizing photons is fairly well determined and our analysis has deduced the ionization parameters which characterize these regions (see Davidson and Netzer 1979). The BLR clouds are 
found to occur at a radius of

$$
R_{\mathrm{BLR}}=4.1 \times 10^{16}\left(\frac{N}{10^{9.5} \mathrm{~cm}^{-3}}\right)^{-1 / 2} \mathrm{~cm},
$$

roughly an order of magnitude larger than the size of the central engine as deduced from variability arguments (see Mushotzky, Holt, and Serlemitsos 1978), and in good agreement with the distance inferred from the time. lag between continuum and emission line variations (Lyuti 1977). The NLR clouds have a much lower ionization parameter and density which corresponds to a radius

$$
R_{\mathrm{NLR}}=9.6 \times 10^{19}\left(\frac{N}{10^{3.5} \mathrm{~cm}^{-3}}\right)^{-1 / 2} \mathrm{~cm} .
$$

At a distance of $20 \mathrm{Mpc}$ this radius will have a full angular size of

$$
\theta_{\mathrm{NLR}}=0.7\left(\frac{N}{10^{3.5} \mathrm{~cm}^{-3}}\right)^{-1 / 2} \operatorname{arc~sec},
$$

which is just resolved on the sky, as was found by Ulrich (1973) and by Penston et al. (1981).

If the gas were gravitationally bound to NGC 4151, as would be the case for either systematic orbital motion or a more chaotic virial equilibrium situation, then these radii would be related to the Doppler widths of the emission lines by $v \propto r^{-1 / 2}$. In such a case the ratio of BLR and NLR line widths would be

$$
\begin{aligned}
\frac{\mathrm{FWHM}_{\mathrm{BLR}}}{\mathrm{FWHM}_{\mathrm{NLR}}} & =\left(\frac{R_{\mathrm{BLR}}}{R_{\mathrm{NLR}}}\right)^{-1 / 2} \\
& =48\left[\frac{N_{\mathrm{BLR}}}{10^{9.5} \mathrm{~cm}^{-3}}\right]^{1 / 4}\left[\frac{N_{\mathrm{NLR}}}{10^{3.5} \mathrm{~cm}^{-3}}\right]^{-1 / 4} .
\end{aligned}
$$

For comparison, the NLR is observed to have a line width of $\mathrm{FWHM}_{\mathrm{NLR}} \approx 230 \mathrm{~km} \mathrm{~s}^{-1}$ (Heckman et al. 1981); the BLR shows FWHM $_{\text {BLR }} \approx 1900 \mathrm{~km} \mathrm{~s}^{-1}$ (Osterbrock and Koski 1976), which corresponds to $\mathrm{FWHM}_{\mathrm{BLR}} / \mathrm{FWHM}_{\mathrm{NLR}}=8.3$. Apparently NLR clouds are moving $\sim 6$ times too quickly relative to BLR clouds for both to be confined in the same gravitational potential well.

The nature of the true NLR and BLR motions is still unclear, but a systematic velocity field with both under the influence of the same gravitational mass seems to be ruled out. If the BLR clouds are rather radiatively accelerated away from the central object and are not bound to the system, then we can set an upper limit to the mass of the central object from

$$
M<\frac{g_{\mathrm{rad}} R_{\mathrm{BLR}}^{2}}{G} \approx 1.8 \times 10^{7} M_{\odot} .
$$

(Since the observed bolometric luminosity of $\sim 10^{44}$ ergs $s^{-1}$ corresponds to an Eddington mass of only $\sim 10^{5.6} M_{\odot}$ the system is likely to be sub-Eddington, as was previously suggested by Mushotzky 1982 from arguments based on the origin of the X-ray variability. BLR clouds are still driven outward because of the increased photoelectric opacity of the gas). Since the escape velocity for a mass of $10^{7} M_{\odot}$ is only $\sim 58 \mathrm{~km} \mathrm{~s}^{-1}$ at the radius of the NLR, while the lines show FWHM $\approx 230$ $\mathrm{km} \mathrm{s} \mathrm{s}^{-1}$, it appears that either the NLR gas is not confined to the system (which would be in keeping with the ideas of Heckman et al. 1981) or that a very large mass $\left(\sim 2 \times 10^{8} M_{\odot}\right)$ is present within the NLR radius.

\section{b) Column Densities}

Table 2 summarizes the predicted BLR and NLR column densities of several ionization species. Only the five lowest stages of ionization are listed since the table is intended to facilitate comparison with optical and ultraviolet spectral line observations.

The sizable differences between these column densities and those found by Penston et al. (1981) are due to our different assumptions concerning the nature of the large widths of the absorption lines. As outlined in $\S$ II $c$, we assume that the widths are due to relative motion of many different clouds, each having only thermal broadening, while Penston et al. attribute the widths to an internal Mach 100 microturbulence.

We note several implications of these column densities. First, the broad line region is the dominant source of X-ray extinction. Table 2 shows that the column density through the BLR gas is $\sim 56$ times larger than through NLR gas. For plane parallel geometry it is easily shown that the column density varies as $U$;

$$
N L \sim \frac{U c}{\alpha} \approx 1.1 \times 10^{23} U \mathrm{~cm}^{-2}
$$

where $\alpha$ and $c$ are the recombination coefficient and speed of light, respectively. Clouds with largest $U$ will tend to dominate extinction of soft X-rays. Although soft X-ray observations primarily contain information concerning the BLR, as was assumed by Holt et al. (1980), Lyman limit observations could determine whether NLR clouds also lie along the line of sight. If only BLR clouds extinguish the continuum, then the Lyman limit will only suffer the $\sim 90 \%$ absorption as is shown in Figure 1. However, NLR clouds also have a sufficient column to fully extinguish Lyman limit radiation, so observations of this spectral region could determine whether NLR clouds fully cover the source, as we predict. Such a measurement could also have implications regarding the continuous extinction of the nucleus, since it seems likely that the NLR gas also contains dust.

Another interesting result of our calculations is that BLR clouds should be opaque to $\lambda>10^{-2} \mathrm{~cm}$ radio 
TABLE 2

Log (COLumn Densities)

\begin{tabular}{|c|c|c|c|c|c|c|}
\hline Element & Region & I & II & III & IV & $\mathrm{V}$ \\
\hline \multirow[t]{2}{*}{ Hydrogen .... } & BLR & 20.16 & 22.99 & $\ldots$ & $\ldots$ & $\ldots$ \\
\hline & NLR & 21.12 & 20.65 & $\ldots$ & $\ldots$ & $\ldots$ \\
\hline \multirow[t]{2}{*}{ Helium ........ } & BLR & 21.73 & 21.49 & 21.11 & $\ldots$ & $\ldots$ \\
\hline & NLR & 19.83 & 19.95 & 19.29 & $\ldots$ & $\ldots$ \\
\hline \multirow[t]{2}{*}{ Carbon ....... } & BLR & 14.65 & 19.46 & 18.60 & 18.84 & 18.42 \\
\hline & NLR & 12.59 & 17.22 & 16.88 & 15.64 & 15.50 \\
\hline \multirow[t]{2}{*}{ Nitrogen ...... } & BLR & 15.37 & 18.77 & 18.05 & 18.14 & 17.17 \\
\hline & NLR & 15.90 & 16.59 & 15.58 & 14.86 & 14.42 \\
\hline \multirow{2}{*}{ Oxygen ....... } & BLR & 16.59 & 19.68 & 19.11 & 18.90 & 18.49 \\
\hline & NLR & 17.56 & 16.53 & 16.51 & 15.74 & 15.77 \\
\hline \multirow{2}{*}{ Neon.......} & BLR & 18.52 & 18.36 & 18.42 & 17.69 & 17.60 \\
\hline & NLR & 14.31 & 15.84 & 16.68 & 15.07 & 14.82 \\
\hline \multirow[t]{2}{*}{ Magnesium ... } & BLR & 14.24 & 18.08 & 17.83 & 17.21 & 17.03 \\
\hline & NLR & 11.61 & 15.26 & 16.06 & 14.43 & 13.95 \\
\hline \multirow[t]{2}{*}{ Silicon.....} & BLR & 11.89 & 17.23 & 18.28 & 17.59 & 17.83 \\
\hline & NLR & 10.93 & 16.28 & 14.28 & 14.85 & 14.74 \\
\hline \multirow[t]{2}{*}{ Sulphur ...... } & BLR & 13.62 & 18.16 & 17.67 & 17.56 & 17.61 \\
\hline & NLR & 10.82 & 15.32 & 16.14 & 14.80 & 14.24 \\
\hline \multirow[t]{2}{*}{ Argon ......... } & BLR & 17.00 & 17.23 & 17.09 & 17.02 & 16.45 \\
\hline & NLR & 12.69 & 14.35 & 15.44 & 13.98 & 13.48 \\
\hline \multirow[t]{2}{*}{ Iron...$\ldots \ldots$} & BLR & 12.91 & 17.92 & 17.19 & 17.48 & 17.08 \\
\hline & NLR & 10.79 & 15.46 & 14.35 & 14.78 & 14.85 \\
\hline
\end{tabular}

emission. The inverse bremsstrahlung optical depth through our BLR cloud is

$$
\begin{aligned}
\tau & =0.18 g_{\mathrm{gg}} T^{-3 / 2} \nu^{-2} N^{2} L \\
& \approx 6.6 \times 10^{24} \nu^{-2}\left(\frac{N}{10^{9.5} \mathrm{~cm}^{-3}}\right) .
\end{aligned}
$$

Since the brightness temperature of BLR clouds is much lower than that of the continuum source, we expect to see a dramatic low-frequency turnover at $\nu \sim 2.6 \times 10^{12}$ $\mathrm{Hz}$. Just such a turnover is in fact seen (see Beall et al. 1981) and should be monitored to obtain the same type of kinematic and spatial information as has been deduced from the X-ray turnover. We note that the class of low luminosity Seyfert 1 galaxies has anomalously weak compact radio components (see de Bruyn and Wilson 1978; de Bruyn 1981). Since this class also has a large covering factor (Mushotzky 1982), GHz radio emission from the central object should be strongly attenuated by intervening BLR clouds.

\section{c) Variability}

Two types of variability are present in NGC 4151. Both the continuum luminosity (see Mushotzky, Holt, and Serlemitsos 1978) and emission-line and continuum absorption strengths (see Barr et al. 1977; Anderson 1974) change over short time scales. It has been unclear how or whether these changes are correlated.

We find that sudden changes in the ionizing flux, such as the flarelike event observed by Mushotzky, Holt, and
Serlemitsos (1978), will produce observable changes in strengths of absorption lines but not in the X-ray absorption. Basically the effect of such a flare is to temporarily increase the ionization parameter of the gas. Line-of-sight BLR clouds can respond to such changes on the recombination time scale of the gas,

$$
\tau_{\mathrm{rec}} \approx\left(N_{e} \alpha_{A}\right)^{-1} \approx 13\left(\frac{N}{10^{9.5} \mathrm{~cm}^{-3}}\right)^{-1} \text { minutes }
$$

i.e., they follow the variation closely. Since light travel time delays do not affect the absorption component, we would predict that individual line optical depths should follow Figure 3 quite closely. If the deduced value of the BLR ionization parameter represents a time average of the continuum flux, then the flare observed by Mushotzky, Holt, and Serlemitsos (1978) would correspond to an increase in $U$ of $\approx 0.25 \mathrm{dex}$, or from $U=10^{-0.75}$ to $10^{-0.50}$. Figure 3 shows that, as a result of this change, the optical depth of both the Lyman and Balmer continuua and lines decrease dramatically; the $\mathrm{C}$ IV $\lambda 1549$ depth increases; while the $\mathrm{He}$ I $\lambda 3889, \mathrm{Mg}$ II $\lambda 2798$, and $N$ v $\lambda 1240$ optical depths remain roughly constant. For comparison, Anderson (1974) noted that large fluctuations in the absorption component of Balmer lines were observed, while the He I $\lambda 3889$ line remained nearly constant. Penston et al. (1981) also note several absorption line-continuum correlations which are in general agreement with our predictions. Careful observational studies of the behavior of absorption lines during flares could provide an empirical measure of the 
slope of the $\tau-U$ curves and provide an important observational test of the validity of BLR model calculations.

Although changes in the luminosity in ionizing radiation will affect optical and ultraviolet absorption lines, tests show that these changes will not affect the X-ray absorption for clouds of constant thickness. Basically, the heavy elements whose inner electrons provide the dominant opacity source for soft X-rays all have their $\mathrm{K}$ shells filled for ionization parameters in the range $10^{-1}$ $<U<10^{\circ}$, and no change in the X-ray opacity occurs (although helium is an exception). The change in the column density must be due to random motions of clouds across the line of sight, as suggested by Holt et al. (1980).

\section{CONCLUSIONS}

We reach the following conclusions regarding the absorbing material in NGC 4151;

1. The absorption arises mainly in a large number of small $\left(\delta r \sim 10^{13.5} \mathrm{~cm}\right)$ clouds which lie a distance $\sim 4 \times$ $10^{16} \mathrm{~cm}$ from the central object. These clouds have a covering factor near unity and an ionization parameter $\sim 30$ times larger than typical QSO clouds. Predictions of model calculations are in substantial agreement with the observed spectrum.

2. The outer NLR clouds are likely to be heated by the continuum as modified by BLR absorption. Models with $\mathrm{H}$ II region abundances, a density of $10^{3.5} \mathrm{~cm}^{-3}$, a distance of $9.6 \times 10^{19} \mathrm{~cm}$, and nearly full coverage are in good agreement with observations. The NLR gas does not give rise to an appreciable amount of extinction of hard radiation.

3. The BLR clouds are likely to be in a radiatively driven wind. The fact that virtually the entire blue half of Ly $\alpha$ is absent and that discrete absorption components always lie shortward of the systemic velocity shows that the gas is in outflow, and our model calculations show the BLR clouds in NGC 4151 can probably be accelerated coherently. The clouds are more likely to resemble biscuits or dumplings rather than pancakes, however.
4. Dust cannot survive in the BLR gas but seems likely to be present in NLR clouds. Emission from dust in NLR clouds is not sufficient to account for the observed infrared flux. The infrared emission is too large to be the result of reprocessed ultraviolet and $\mathrm{X}$-ray radiation since our analysis shows that most of this energy has gone into emission-line cooling.

5. BLR clouds should be opaque to radio waves. We show that, for the deduced physical conditions, BLR clouds are thick to inverse bremsstrahlung for wavelengths longer than $\sim 10^{-2} \mathrm{~cm}$. This predicted turnover may have already been detected.

6. We predict several correlations between continuum luminosity and line optical depths. Observations of absorption lines during periods of continuum variability could provide an important test of model calculations.

7. There does not exist any additional source of ionizing radiation other than the extrapolated ultraviolet and $\mathrm{X}$-ray continua. This follows from the energy budget of the BLR, the covering factor deduced from the X-ray absorption, and the observed continua.

The need for more observations of NGC 4151 is obvious. The behavior of the absorption lines as a function of time is particularly important since this provides kinematic and spatial information available in no other way. High-resolution observations of these lines with good signal-to-noise may actually resolve individual clouds within the broad feature. Accurate emission-line profile studies would also be beneficial since NGC 4151 is likely to be a case of outflow and photoionization models predict that several types of line asymmetries should occur. All these avenues of attack offer important information on physical conditions in one of the few well-documented cases of radiatively driven outflowing gas.

The discussion of the NLR gas was prompted by E. A. Boldt. R. F. M. thanks C.-C. Wu for several informative discussions. G. J. F. thanks the NSF for support through grant AST 80-25222 and acknowledges the hospitality of the Laboratory for High Energy Astrophysics.

\section{REFERENCES}

Adams, T. F. 1972, Ap. J. 174, 439.

Allen, D. A. 1979, M.N.R.A.S., 186, 1P.

Anderson, K. S. 1974, Ap. J., 189, 195.

Anderson, K. S., and Kraft, R. P. 1969, Ap. J., 158, 859.

Baldwin, J. A., 1977a, M.N.R.A.S., 178, 67P. 1977b, Ap. J., 214, 679.

Baldwin, J. A., and Netzer, H. 1978, Ap. J., 226, 1.

Barr, P., White, N., Sanford, P., and Ives, J., 1977, M.N.R.A.S., 181, 43P.

Beall, J., Rose, W., Dennis, B., Crannell, C., Dolan, J., Frost, K., and Orwig, L. 1981, Ap. J., 247, 458.

Blumenthal, G., and Mathews, W., 1975, Ap. J., 198, 517. 1979, Ap. J., 233, 479.

$\overline{B o k s e n}$ berg, A., and Netzer, H. 1977, Ap. J., 212, 37.

Boksenberg, A., and Penston, M. 1976, M.N.R.A.S., 177, 127P.
Boksenberg, A., Shortridge, K., Allen, D. A., Fosbury, R., Penston, M., and Savage, A. 1975, M.N.R.A.S., 173, 381 .

Burgess, A., and Summers, H. 1976, M.N.R.A.S., 174, 345

Canfield, R. C., and Puetter, R. C. 1981, Ap.J., 243, 381

Capriotti, E. R. 1965, Ap. J., 142, 1101.

Capriotti, E. R., Foltz, C. B., and Byard, P. L. 1979, Ap. J., 230, 681.

Carswell, R. F., and Ferland, G. J., 1980, M.N.R.A.S., 191, 55.

Clayton, D., and Wickramasinghe, N. 1976, Ap. Sp. Sci., 42, 463.

Cromwell, R., and Weymann, R. 1970, Ap.J. (Letters), 159, L147.

Cruddace, R., Paresce, F., Bowyer, S., and Lampton, M. 1974, Ap. $J ., 187,497$.

Cutri, R. M., et al. 1981, Ap. J., 245, 818.

Daltabuit, E., MacAlpine, G. M., and Cox, D. P. 1978, Ap. J., 219, 
Davidson, K. 1972, Ap. J., 171, 213. 1973, Ap.J., 181, 1

1975, Ap. J., 195, 285

1977, Ap. J., 218, 20.

Davidson, K., and Netzer, H. 1979, Rev. Mod. Phys., 51, 715. de Bruyn, A. G. 1981, Talk given at the 24th Herstmonceux Conference on Galactic Nuclei.

de Bruyn, A. G., and Wilson, A. S. 1978, Astr. Ap., 64, 433.

Drake, S. A., and Ulrich, R. K. 1980, Ap. J. Suppl., 42, 351.

Fabian, A. C., and Ross, R. R. 1981, M.N.R.A.S., 195, 29P.

Ferland, G. J. 1981, Ap. J., 249, 17.

Ferland, G. J., and Netzer, H. 1979, Ap. J., 229, 274. 1982, Ap. J., in press.

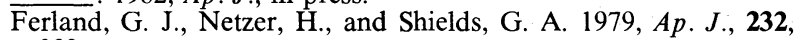
382.

Ferland, G. J., and Truran, J. W. 1981, Ap. J., 244, 1022.

Fosbury, R., Mebold, U., Goss, W., and Dopita, M. 1978, M.N.R.A.S., 183, 549.

French, H. B. 1980, Ap. J., 240, 41.

Heckman, T. M., Miley, G. K., van Breugel, W., and Butcher, H. 1981, Ap. J., 247, 403.

Holt, S. S., Mushotzky, R. F., Becker, R. H., Boldt, E. A., Serlemitsos, P., Szymkowiak, A. E., and White, N. E. 1980, Ap. J. (Letters), 241, L13.

Kaler, J. B. 1978, Ap. J., $220,887$.

Krolik, J., McKee, C. F., and Tarter, C. B. 1981, Ap. J., 249, 422.

Kwan, J., and Krolik, J., 1981, Ap. J., 250, 478.

Lambert, D. L. 1978, M.N.R.A.S., 182, 249.

Lambert, D. L., and Luck, R. E. 1978, M.N.R.A.S., 183, 79.

Lyuti, Z. M., 1977, Soviet Astr.- AJ, 21, 655.

MacAlpine, G. M. 1972, Ap. J., 175, 11. . 1981, Ap. J., 251, 465.

Malkan, M. A., and Sargent, W. L. W. 1982, Ap. J., 254, 22.

Martin, P. G., and Ferland, G. J. 1980, Ap. J. (Letters), 235, L125.

Mushotzky, R., Holt, S. S., and Serlemitsos, R. 1978, Ap. J. (Letters), 225, L115.
Mushotzky, R. F., Marshall, F. E., Boldt, E. A., Holt, S. S., and Serlemitsos, R. 1980, Ap. J., 235, 377.

Mushotzky, R. 1982, Ap. J., 256, 92.

Netzer, H. 1974, M.N.R.A.S., 169, 579. . 1980, Ap. J., 236, 406. 1982, M.N.R.A.S 198,589

Netzer, H., and Davidson, K., 1979, M.N.R.A.S., 187, 871.

Osterbrock, D. E. 1974, Astrophysics of Gaseous Nebulae (San Francisco: Freeman). . 1977, Ap. J., 215, 733.

Osterbrock, D. E., and Koski, A. T. 1976, M.N.R.A.S., 176, 61P.

Penston, M. V., Clavel, J., Snijders, M., Boksenberg, A., and Fosbury, R. 1979, M.N.R.A.S., 189, 45P.

Penston, M. V., et al. 1981, M.N.R.A.S., 196, 857.

Peterson, B. M., Foltz, C. B., Capriotti, E. R., and Wu, C.-C. 1982, preprint.

Savage, B. D., and Bohlin, R. C. 1979, Ap. J., 229, 136.

Seaton, M. J. 1978, M.N.R.A.S., 185, 5P.

Shields, G. A. 1974, Ap. J., 191, 309.

Shields, G. A., and McKee, C. F. 1981, Ap. J. (Letters), 246, L57.

Shields, G. A., and Mushotzky, R. 1979, Astr. Ap., 79, 56

Shuder, J. M., and MacAlpine, G. M. 1979, Ap. J., 230, 348.

Shull, J. M. 1979, Ap. J., 234, 761.

Smith, M., et al. 1981, M.N.R.A.S., 195, 437.

Storey, P. 1981, M.N.R.A.S., 195, 27P.

Summers, H. P. 1974, Appleton Lab Internal Memo 367.

Ulrich, M. H. 1973, Ap. J., 181, 51.

Weisheit, J., Shields, G., and Tarter, C. 1981, Ap. J., 245, 406.

Weymann, R., and Williams, R. 1969, Ap.J., 157, 1201.

Williams, R. E. 1967, Ap. J., 147, 556.

Wills, B. J., Netzer, H., and Wills, D. 1980, Ap. J. (Letters), 242,

Wilson, A. S., 1979, Proc. Royal Soc. London A, 366, 461.

G. J. FERLAND: Department of Physics and Astronomy, University of Kentucky, Lexington, KY 40506

RichaRd Mushotzky: Laboratory for High Energy Astrophysics, Code 661, NASA Goddard Space Flight Center, Greenbelt, MD 20771 